\title{
Aborted sternotomy due to unexpected porcelain aorta: Does transcatheter aortic valve replacement offer an alternative choice?
}

\author{
Jahanzaib Idrees, MD, ${ }^{\mathrm{a}}$ Eric E. Roselli, MD, ${ }^{\mathrm{a}}$ Sajjad Raza, MD, ${ }^{\mathrm{a}}$ Amar Krishnaswamy, MD, \\ Stephanie Mick, MD, ${ }^{\mathrm{a}}$ Samir Kapadia, MD, ${ }^{\mathrm{b}}$ Gosta B. Pettersson, MD, PhD, ${ }^{\mathrm{a}}$ Murat Tuzcu, MD, ${ }^{\mathrm{b}}$ and \\ Lars G. Svensson, MD, $\mathrm{PhD}^{\mathrm{a}}$
}

\begin{abstract}
Objectives: Surgical aortic valve replacement is challenging in patients with severe aortic calcification. Some patients undergo sternotomy and have the operation aborted because of intraoperative discovery of severe calcification. Hypothermic circulatory arrest and transcatheter aortic valve replacement offer clampless treatment options for aortic stenosis. The study objectives are to characterize patients who are referred after sternotomy was aborted for porcelain aorta and to describe the treatment outcomes.
\end{abstract}

\begin{abstract}
Methods: From 2001 to 2013, 19 patients presented after attempt at surgical aortic valve replacement was aborted because of porcelain aorta. Patients presented with aortic stenosis $(n=16)$, regurgitation $(n=1)$, or both $(\mathrm{n}=2)$. Off-pump coronary bypass was performed in 10 patients. At the Cleveland Clinic, patients underwent surgical aortic valve replacement $(n=7)$ or transcatheter aortic valvve replacement $(n=12)$. The median interval between aborted aortic valve replacement and definitive treatment was 9.6 months. The mean age was 74 \pm 11 years. The mean transvalvular gradient was $51 \pm 18 \mathrm{~mm} \mathrm{Hg}$, and area was $0.6 \mathrm{~cm}^{2}$. Axillary cannulation was used in all patients undergoing surgical aortic valve replacement, but only 4 required circulatory arrest. The transcatheter aortic valve replacement approach was transfemoral $(n=5)$, transapical $(n=6)$, or transaortic $(\mathrm{n}=1)$.
\end{abstract}

Results: The mean postoperative gradient was $13 \pm 4 \mathrm{~mm} \mathrm{Hg}$. There was no mortality, stroke, renal failure, or reoperation for bleeding. One patient required a second valve implantation for paravalvular leak. The median hospital length of stay was 8 days. Five late noncardiac deaths occurred at a median follow-up of 16 months.

Conclusions: Both surgical aortic valve replacement and transcatheter aortic valve replacement are safe and effective options after aborted sternotomy in patients with porcelain aorta who are referred to a high-risk valve center. Procedure selection may be tailored to individual patients on the basis of aortic morphology and comorbidities. Patients with aortic stenosis at risk for calcific aortic disease should be screened with cross-sectional imaging preoperatively. (J Thorac Cardiovasc Surg 2015;149:131-4)

See related commentary on pages 134-6.

Surgical aortic valve replacement (SAVR) is a challenge in patients with porcelain aorta, because the calcified aorta is difficult to manage. Studies have reported significant risks of stroke and mortality in these patients. ${ }^{1}$

The conventional treatment for aortic stenosis is SAVR, but the advent of transcatheter aortic valve replacement (TAVR) has expanded treatment options to operable and

\footnotetext{
From the Department of Thoracic and Cardiovascular Surgery ${ }^{\mathrm{a}}$ and Interventional Cardiology, ${ }^{\mathrm{b}}$ Heart and Vascular Institute, Cleveland Clinic, Cleveland, Ohio.

Disclosures: Eric E. Roselli reports consulting fees from Edwards and Medtronic. All

other authors have nothing to disclose with regard to commercial support.

Received for publication Aug 2, 2014; revisions received Sept 9, 2014; accepted for publication Sept 11, 2014; available ahead of print Nov 4, 2014.

Address for reprints: Eric E. Roselli, MD, Department of Thoracic and Cardiovascu-

lar Surgery, Cleveland Clinic, 9500 Euclid Ave, Desk J4-1, Cleveland, OH 44195-

5108 (E-mail: roselle@ccf.org).

$0022-5223 / \$ 36.00$

Copyright (c) 2015 by The American Association for Thoracic Surgery

http://dx.doi.org/10.1016/j.jtcvs.2014.09.035
}

higher-risk patients. Because TAVR does not require cardiac arrest, the need to clamp the aorta is avoided, but this treatment has been reserved for high-risk patients with suitable anatomy. ${ }^{2}$

Since the advent of TAVR, we have encountered an increase in patients with severe aortic stenosis in whom SAVR was attempted but aborted because of intraoperative discovery of porcelain aorta. These patients were referred for definitive treatment and possible TAVR.

Both SAVR and TAVR were performed in these patients. The objectives are to characterize patients, describe treatment options, and assess outcomes.

\section{PATIENTS AND METHODS}

From 2001 to 2013, 19 patients with a history of aborted SAVR and severe aortic valve dysfunction underwent definitive aortic valve replacement with SAVR $(\mathrm{n}=7)$ or TAVR $(\mathrm{n}=12)$ at the Cleveland Clinic. The mean age at the time of operation was $74 \pm 11$ years. Aortic valve dysfunction included severe aortic stenosis $(n=16)$, regurgitation $(n=1)$, or both $(\mathrm{n}=2)$. The mean preoperative aortic valve gradient was $53 \pm 18 \mathrm{~mm}$ Hg (SAVR: $46 \pm 8$ mm Hg, TAVR: $56 \pm 16 \mathrm{~mm} \mathrm{Hg}$ ). Table 1 summarizes the patient characteristics for both groups. 


\section{Abbreviations and Acronyms}

SAVR $=$ surgical aortic valve replacement

$\mathrm{TAVR}=$ transcatheter aortic valve replacement

The median interval between aborted aortic valve replacement at the outside facility and definitive procedure with SAVR or TAVR at the Cleveland Clinic was 9.6 months. In 10 patients (53\%), off-pump coronary artery bypass grafting was performed at the time of aborted aortic valve replacement, and the sternotomy was closed without additional procedures in the remaining 9 patients. Data were collected from chart review and augmented with Social Security Death Index when available. Descriptive statistical analyses are used to present variables for the study. The study was approved by the institutional review board of Cleveland Clinic, with patient consent waived.

\section{Surgical Aortic Valve Replacement}

The surgical approach was via redo sternotomy (median, 6; minimally invasive, 1). Bioprosthetic valves were used in 5 patients, and a mechanical valve was used in 2 patients. Two patients in this group also had bicuspid aortic valve. The median prosthetic valve size was $25 \mathrm{~mm}$. The aorta was not clampable in 4 patients because of severe calcification of the ascending aorta, and hypothermic circulatory arrest with retrograde brain perfusion was used. The ascending aorta was replaced in all 4 patients. In the other 3 patients, the ascending aorta was relatively spared of calcification near the brachiocephalic artery, and it was possible to crossclamp the aorta in that region to avoid circulatory arrest. In no patients was there adequate healthy aorta for both clamping and cannulating. Right axillary artery cannulation was applied in all 7 patients. Nine concomitant procedures were performed in 6 patients for coexisting cardiac disease. These included ascending aortic replacement $(n=4)$, coronary bypass $(n=3)$, mitral valve repair $(n=1)$, and tricuspid valve repair $(n=1)$. One patient with severe radiation heart disease required all 4 procedures along with SAVR.

\section{Transcatheter Aortic Valve Replacement}

This technique was used in 12 patients. The approach was transfemoral in 5 patients. The other 7 patients had severe peripheral arterial disease prohibiting this approach. These were treated using transapical $(n=6)$ or transaortic $(\mathrm{n}=1)$ access. Balloon-expandable valves (Sapien, Edwards

TABLE 1. Preprocedural patient characteristics

\begin{tabular}{|c|c|c|c|}
\hline & Overall & SAVR & TAVR \\
\hline & $\mathbf{N}=19$ & $\mathbf{n}=\mathbf{7}$ & $\mathbf{n}=\mathbf{1 2}$ \\
\hline Age (mean y) & $74 \pm 11$ & $62 \pm 12$ & $78 \pm 3$ \\
\hline Male & 12 & $3(43)$ & $9(75)$ \\
\hline NYHA class II/III & 17 & $6(86)$ & $11(92)$ \\
\hline Hypertension & 13 & $6(86)$ & $7(58)$ \\
\hline COPD & 4 & $1(14)$ & $3(25)$ \\
\hline Cancer & 5 & $2(17)$ & $3(25)$ \\
\hline Prior stroke & 3 & $0(0)$ & $3(25)$ \\
\hline \multicolumn{4}{|l|}{ Cardiac comorbidities } \\
\hline Coronary artery disease & 12 & $7(100)$ & $5(42)$ \\
\hline Ascending aortic aneurysm & 1 & $1(14)$ & $0(0)$ \\
\hline Radiation heart disease & 2 & $1(14)$ & $1(8)$ \\
\hline Mitral valve disease & 1 & $2(28)$ & $0(0)$ \\
\hline Tricuspid valve disease & 1 & $1(14)$ & $0(0)$ \\
\hline
\end{tabular}

COPD, Chronic obstructive pulmonary disease; NYHA, New York Heart Association; SAVR, surgical aortic valve replacement; TAVR, transcatheter aortic valve replacement.
Lifesciences, Santa Rosa, Calif) were used in all patients in this group. Valve sizes were $23 \mathrm{~mm}$ in 4 patients and $26 \mathrm{~mm}$ in 8 patients.

In 1 patient in this group, intraoperative prosthetic paravalvular leak developed that was managed with placement of an additional valve-invalve. Another developed hemodynamic instability and required temporary use of an intra-aortic balloon pump.

\section{RESULTS}

The mean postoperative gradients were $13 \pm 4 \mathrm{~mm} \mathrm{Hg}$ (SAVR: $14 \pm 3$, TAVR: $14 \pm 4 \mathrm{~mm} \mathrm{Hg}$ ). At a median follow-up of 16 months, there was no operative mortality, paravalvular leak, stroke, renal failure, or reoperation for bleeding. There were 5 late deaths, 4 in the TAVR group and 1 after SAVR. The patient who died post-SAVR had lung cancer and died approximately 1 year postoperatively of pneumonia associated with the lung cancer. The remaining 4 deaths in the TAVR group all occurred in patients with multiple comorbidities. One patient died 5 months post-TAVR of persistent heart failure due to diastolic dysfunction and severe mitral and tricuspid regurgitation despite a well-functioning TAVR. One patient died of complications related to respiratory failure 2 months post-transaortic TAVR at a long-term care facility. She had a recent history of lung cancer treated with radiation on continuous oxygen and required a tracheostomy postprocedure. One patient died 17 months post-TAVR of complications related to a chronic gastrointestinal bleed, and 1 patient died 3 years later of acute on chronic renal failure despite a well-functioning TAVR valve. No patients required valve-related reintervention during late follow-up.

\section{DISCUSSION}

The conventional approach for treatment of severe aortic stenosis is SAVR, but the procedure can be technically challenging when the aorta is severely calcified, and this also increases the risk of stroke. ${ }^{1-3}$ Studies have reported a mortality of up to $14 \%$ in these patients. ${ }^{3}$ This experience demonstrates that both SAVR and TAVR can be safely performed at a valve center specializing in the treatment of high-risk patients. TAVR expands options by providing a clampless alternative for replacing the aortic valve in patients with calcified aorta who may be too high risk for SAVR. ${ }^{4-6}$ Use of axillary artery cannulation with or without hypothermic circulatory arrest may facilitate SAVR in these patients who are otherwise candidates for aortic valve replacement.

The choice of procedure type was based on a thorough preoperative assessment to determine the operative risk, anatomic feasibility, and need for additional procedures for cardiac comorbidities. SAVR was the preferred choice in patients with multiple cardiac comorbidities requiring additional procedures, such as coronary bypass, ascending aortic replacement, and mitral or tricuspid valve repair. SAVR also was more often performed during the earlier period of this experience when TAVR was not as readily available. Conversely, TAVR was preferred in patients 

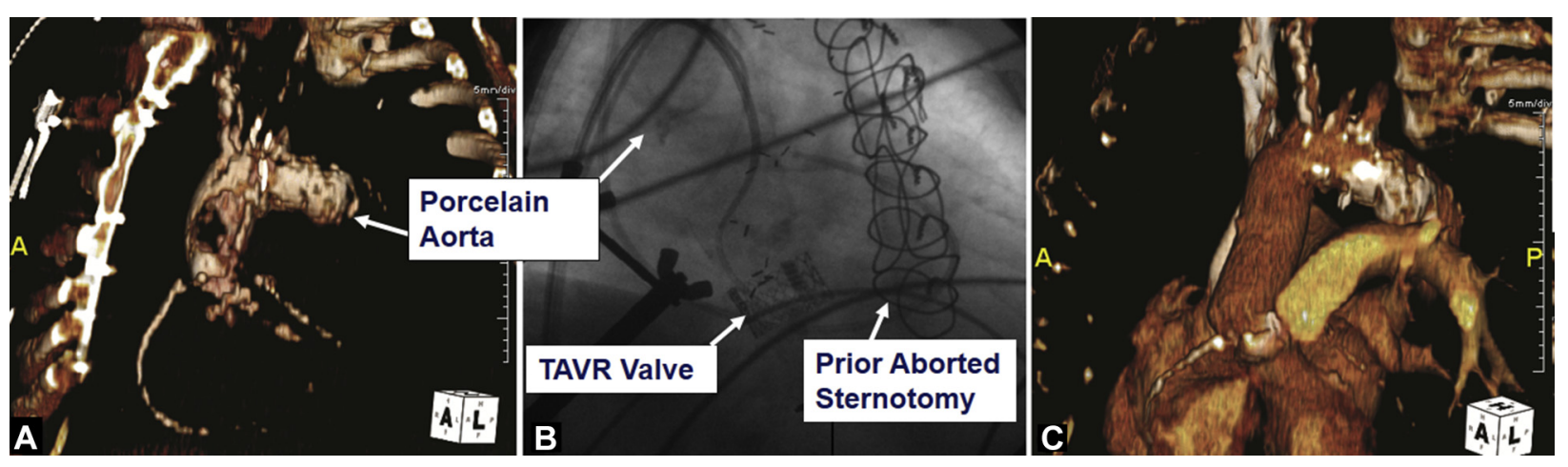

FIGURE 1. A, Preoperative volume-rendered computed tomography image showing severely calcified ascending aorta and arch. B, Periprocedural fluoroscopy image showing sternal wires from previous aborted sternotomy, TAVR valve, and porcelain aorta. C, Postoperative volume-rendered computed tomography image showing bioprosthetic aortic valve and surgical graft after ascending aortic repair. TAVR, Transcatheter aortic valve replacement.

with isolated aortic valve disease and suitable anatomy who did not require additional procedures and those who were too high risk for an open operation.

Previous studies have reported a high incidence of stroke with SAVR in patients with porcelain aorta. In the current experience, this was not observed in any of the patients. At the Cleveland Clinic, we routinely use axillary artery cannulation and have a low threshold to use this technique in patients with aortic disease. This strategy has been shown to decrease the risk of stroke by minimizing manipulation of the atherosclerotic ascending aorta. ${ }^{3,7-9}$ The axillary artery cannulation was used in all patients who were treated with SAVR in this experience.

Most patients treated with SAVR $(n=4 / 7)$ had a diffusely calcified proximal aorta involving the root, ascending aorta, and often extending into the arch and beyond. Careful evaluation of the proximal aorta using 3-dimensional reconstructions was critical in the preoperative planning to determine whether it was possible to crossclamp the aorta (Figure 1). If not, the repair was performed using hypothermic circulatory arrest or a TAVR approach. In patients who are otherwise at a reasonable risk, replacing the ascending aorta in this setting has been shown to decrease the risk of stroke. ${ }^{3}$ The circulatory arrest time is short in these patients because it is essentially used for just a single open distal anastomosis. We have not tended to use antegrade brain perfusion in these patients because it has not been shown to be superior for short periods of circulatory arrest; in addition, the arch branch vessels are typically diseased in these patients, and we have not wanted manipulate them. In 3 patients ( $n=3 / 7$ ), the aortic calcification was found to be patchy on computed tomography, and it was possible to successfully clamp the ascending aorta where it was relatively spared. In these patients, ascending repair was not performed, but axillary cannulation was still used.

In comparison, the patients who were treated with TAVR were older with significant comorbidities and presented with severe aortic valve stenosis and porcelain aorta, but they did not require other cardiac intervention. Five of these patients had coexisting coronary artery disease, but had undergone off-pump coronary artery bypass grafting at the time of prior aborted sternotomy or had been adequately revascularized several years before presenting to the Cleveland Clinic. TAVR expands the options in high-risk patients, many of whom are not candidates for SAVR. In this experience, the median Society of Thoracic Surgeons score was 8 for the patients who underwent TAVR. Although these procedures were all feasible, proper patient selection is critical to successful long-term outcomes. Two of the 5 late deaths occurred within the first 6 months postprocedure. One death was due to respiratory failure in a patient with severe lung disease who had transaortic delivery through a mini-J sternotomy. One late death was due to heart failure related to severe atrioventricular valve dysfunction and diastolic dysfunction. In retrospect, these patients may have been better relegated to medical therapy only as "cohort C" patients.

The choice of delivery for TAVR was based on iliofemoral vessel anatomy. The transfemoral approach was preferred when the anatomy was suitable; otherwise, alternative approaches were used. TAVR offers a distinct advantage in patients with porcelain aorta by alleviating the need for aortic clamping, but it is a complex operation requiring a multidisciplinary skill set. ${ }^{10}$ Many patients with porcelain aorta have vasculopathy, and so only 5 of 12 were amenable to transfemoral delivery. TAVR and SAVR should be considered complementary options for these complex cases, and the best option is determined by the heart team.

\section{CONCLUSIONS}

Both SAVR and TAVR at a tertiary care center are safe and effective treatment options in patients with a severely calcified aorta. Procedure selection may be tailored to individual patients on the basis of anatomy, comorbidities, and perceived operative risk. Preferably, severe aortic disease should be identified and imaged in detail before the aortic valve procedure. 


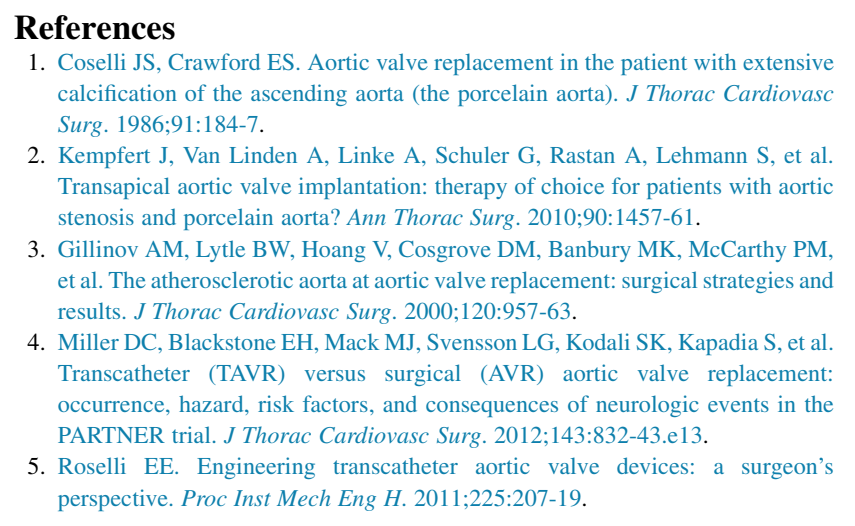

6. Kodali SK, Williams MR, Smith CR, Svensson LG, Webb JG, Makkar RR, et al. Two-year outcomes after transcatheter or surgical aortic-valve replacement. $N$ Engl J Med. 2012;366:1686-95.

7. Sabik JF, Lytle BW, McCarthy PM, Cosgrove DM. Axillary artery: an alternative site of arterial cannulation for patients with extensive aortic and peripheral vascular disease. J Thorac Cardiovasc Surg. 1995;109:885-91.

8. Sabik JF, Nemeh H, Lytle BW, Blacksone EH, Gillinov AM, Rajeswaran J, et al. Cannulation of the axillary artery with a side graft reduces morbidity. Ann Thorac Surg. 2004;77:1315-20.

9. Svensson LG, Blackstone EH, Rajeswaran J, Sabik JF 3rd, Lytle BW, GonzalezStawinski G, et al. Does the arterial cannulation site for circulatory arrest influence stroke risk? J Thorac Cardiovasc Surg. 2000;120:957-63.

10. Roselli EE, Idrees J, Mick S, Kapadia S, Tuzcu M, Svensson LG, et al. Emergency use of cardiopulmonary bypass in complicated transcatheter aortic valve replacement: Importance of a heart team approach. J Thorac Cardiovasc Surg. January 15, 2014 [Epub ahead of print].

\title{
EDITORIAL COMMENTARY
}

\section{Individualized treatment strategies for patients with aortic valve disease and porcelain aorta}

\author{
Scott A. LeMaire, MD
}

See related article on pages 131-4.

The presence of porcelain aorta, defined as severe circumferential calcification of the aorta (Figure 1), substantially increases the complexity and risk associated with the treatment of aortic valve (AV) disease. The unique report by Idrees and colleagues ${ }^{1}$ focuses on a series of 19 patients who were referred for treatment after an attempt at open surgical AV replacement (AVR) was abandoned due to the unanticipated discovery of porcelain aorta. Importantly, this is a contemporary series that began in 2001 and includes the period just before the availability of transcatheter aortic valve replacement (TAVR) devices. The report indicates that excellent early results can be achieved when an individualized approach is applied at an experienced center. Notably, no patients died within 30 days and no patients experienced stroke. Although the report focuses on patients referred after aborted AVR, the

From the Division of Cardiothoracic Surgery, Michael E. DeBakey Department of Surgery, Cardiovascular Research Institute, Baylor College of Medicine, and Department of Cardiovascular Surgery, Texas Heart Institute, Houston, Tex. Disclosures: Author has nothing to disclose with regard to commercial support. Received for publication Oct 4, 2014; accepted for publication Oct 6, 2014

Address for reprints: Scott A. LeMaire, MD, 1 Baylor Plaza, BCM 390, Houston, TX 77030 (E-mail: slemaire@bcm.edu).

J Thorac Cardiovasc Surg 2015;149:134-6

$0022-5223 / \$ 36.00$

Copyright (c) 2015 by The American Association for Thoracic Surgery

http://dx.doi.org/10.1016/j.jtcvs.2014.10.024 results and their implications are relevant to all patients with porcelain aorta and AV disease. Their experience highlights that a successful individualized treatment strategy hinges on several key decision points.

\section{WHETHER TO PERFORM ANY PROCEDURE}

\section{AT ALL}

The first decision point, whether or not to proceed with any AVR intervention, is perhaps the most challenging. In the series by Idrees and colleagues, ${ }^{1}$ there were 2 deaths within 6 months after TAVR; both deaths were due to comorbid conditions, and the authors acknowledge that nonoperative management may have been a better choice for these patients. As articulated by Miller, ${ }^{2}$ the line between utility and futility for TAVR has not yet been clearly defined. Improving our ability to identify those patients who are unsuitable for either open AVR or TAVR remains an extremely important focus of investigation. A recent analysis of Placement of Aortic Transcatheter Valve (PARTNER) trial results to identify predictors of poor outcomes is an excellent step in this direction. ${ }^{3}$

\section{WHETHER TO PERFORM OPEN AVR OR TAVR}

Once the decision has been made to proceed with an intervention, the next decision centers on whether to perform open AVR or TAVR. In the series by Idrees and colleagues, ${ }^{1}$ this decision was largely driven by whether additional cardiac procedures were needed; patients with aneurysmal ascending aorta, multivalve disease, or 